\title{
OPTICAL FREQUENCIES IN V471 TAU
}

\author{
D. E. Winget* and C. F. Claver \\ McDonald Observatory and Department of Astronomy, \\ The University of Texas at Austin
}

$V 471 T a u$ is a spectroscopic and eclipsing binary system located in the Hyades cluster. The binary consists of a $K 2 \mathrm{~V}$ and a hot DA white dwarf star (Nelson and Young 1970). Soft x-ray observations reveal strong modulation at periods of $554.7 \pm 0.3 \mathrm{~s}$ and $277.5 \pm 0.1 \mathrm{~s}$ (Jensen 1985, Jensnen et al. 1986).

Robinson et al. (1988) reported the detection of the $555 \mathrm{~s}$ period in the optical. This period was about a factor of 20 reduced in mean amplitude in their data relative to the soft $x$-ray amplitude. They also found that it varied in amplitude from run to run by more than a factor of 2.5 , dropping below detectability on several runs. They found some evidence for the $277.5 \mathrm{~s}$ period in several runs, but never at high enough amplitude to measure reliably. In addition, they noted that several of their runs had statistically significant power at other frequencies, but noted that in their six runs none of the additional frequencies repeated themselves. Robinson et al. also used observations near the eclipse of the white dwarf to demonstrate that most of the pulsed light is coming from the white dwarf.

We report on optical high speed-photometric observations of the system ( Table 1). Our observations were made in the Strömgren $u$ band in order to minimize the contribution of the K2V star. All of our data were taken on the $2.1 \mathrm{~m}$ telescope at the University of Texas' McDonald Observatory on Mt. Locke, using the Nather 2-star photometer system. The data were reduced as described in Hine (1988).

Journal of Observations: High-Speed Photometry ${ }^{1}$

\begin{tabular}{ccccc}
\hline $\begin{array}{c}\text { Run } \\
\text { Number }\end{array}$ & $\begin{array}{c}\text { Date } \\
(\mathrm{UT})\end{array}$ & $\begin{array}{c}\text { Run Start } \\
(\mathrm{UT})\end{array}$ & $\begin{array}{c}\text { Length } \\
(\mathrm{sec})\end{array}$ & $\begin{array}{c}\text { Telescope } \\
(\mathrm{m})\end{array}$ \\
\hline DEW25 & 17 Feb. 1988 & $01: 50: 43$ & 15450 & 2.1 \\
DEW28 & 18 Feb. 1988 & $02: 11: 40$ & 12100 & 2.1 \\
DEW38 & 22 Feb. 1988 & $02: 09: 50$ & 11510 & 2.1 \\
\hline
\end{tabular}

Table 1

We have detected both frequencies found in the $\mathrm{x}$-ray: the $554 \mathrm{~s}$ period (first detected in the optical by Robinson et al. 1988) and the $277 \mathrm{~s}$ period. Both periods vary significantly in amplitude from night to night; during our runs the $277 \mathrm{~s}$ harmonic had the largest amplitude. Its fractional semi-amplitude varies from $6 \times 10^{-4}$ to $1.2 \times 10^{-3}$. We have found evidence for a number of additional frequencies with statistically significant amplitudes in individual runs and at least one frequency $(P=410 s)$ which is present in all three runs at constant-albeit low-fractional semi-amplitude of $4.5 \times 10^{-4}$.

* Alfred P. Sloan Fellow

1 Data taken with Nather 2-star photometer (cf. Nather, R. E., 1973, Vistas in Astronomy,15, 91). 

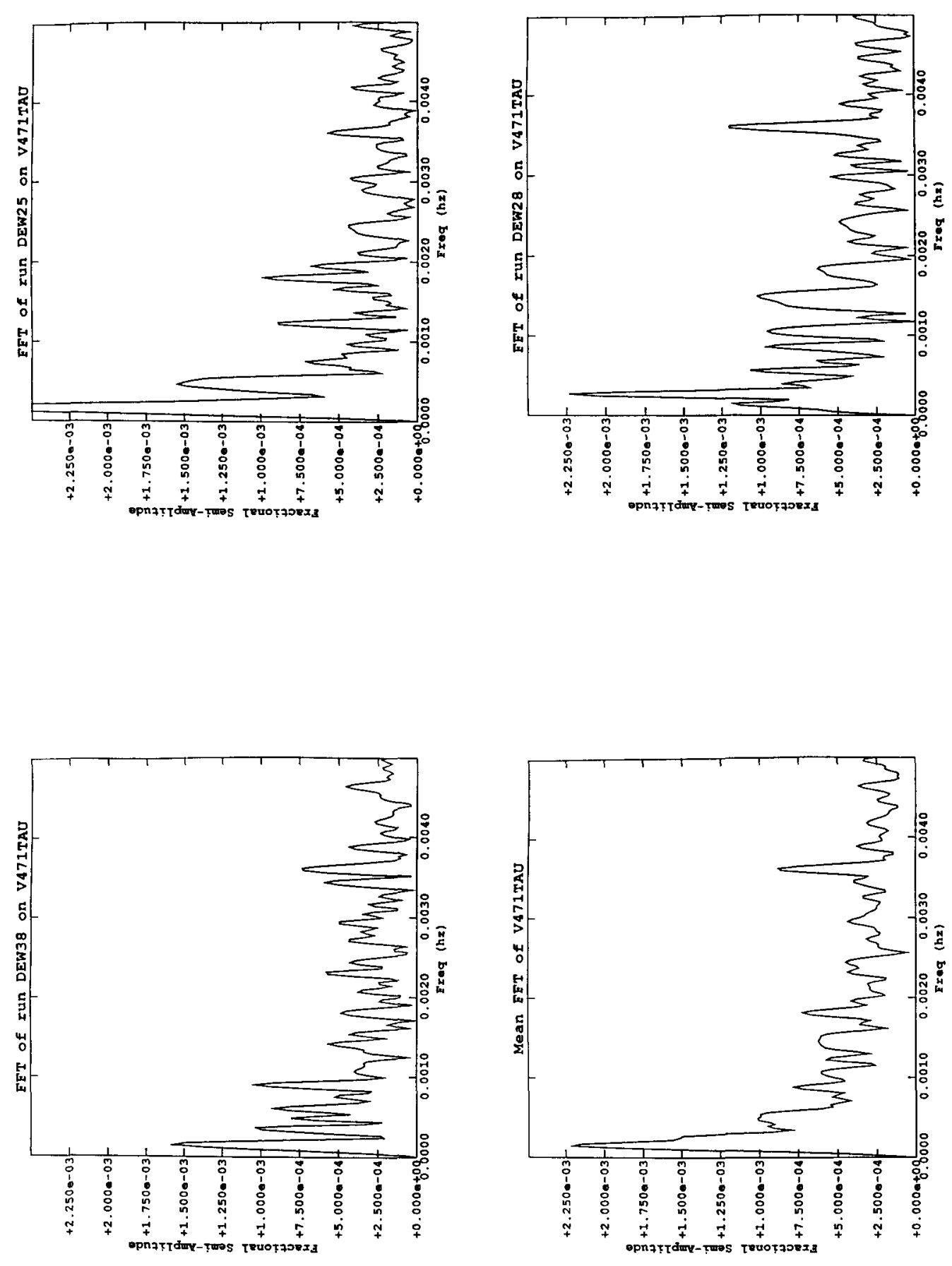
As pointed out by Robinson et al., the presence of peaks in the power spectra in addition to the $555 \mathrm{~s}$, and the $277.5 \mathrm{~s}$ peaks, implies nonradial pulsations of the white dwarf. Our observations provide strong evidence that at least some of the frequencies observed in the white dwarf component are due to nonradial pulsations. However, these observations do not exclude the possibility that the $554 \mathrm{~s}$ or the $277 \mathrm{~s}$ modulations are due to dark/bright spots resulting from wind accretion on a rotating magnetic white dwarf.

We introduce the possibility that the nonradial pulsations may be Rossby modes instead of, or in addition to, the g-modes suggested by Robinson et al. Also, we point out that the excitation mechanism for the modes may be external-the resuit of the wind in the K2 star, or due to a theoretically expected hot DA instability strip.

In principle it is possible, with long uninterrupted data sets, to determine which, if any, frequencies are r-modes, which are g-modes, and which come from possible dark/bright spots on the white dwarf. For example, recall that we observe the amplitude of each of the optical periods to be variable over timescales less than or of order a day. If the period disappears and then reappears with a different phase, it cannot be due to rotation-it must be due to pulsation. Whether the pulsation is an r-mode or $\mathrm{g}$-mode can be determined by the spacing between it and the other periods: r-modes have integer period ratios, while g-modes in general do not. If a period maintains its phase as its amplitude changes, it could be due to pulsation or rotation; however, if it is a g-mode pulsation the phase coherence implies that the amplitude modulation is due to the presence of closely spaced frequencies corresponding to different $\mathrm{m}$-values. Finally, if the excitation is external and the amplitude modulation is caused by fluctuations in the wind from the $\mathrm{K} 2 \mathrm{~V}$ star, then the periods will not maintain phase.

In order to resolve these questions we are currently planning extended time- base observations using the Whole Earth Telescope (cf. Nather 1988, these proceedings) during November 1988.

This work was supported in part by grants, AST 85-52457 and AST 86-00507, from the National Science Foundation.

\section{References}

Hine, B. P. A., III, 1988, A Seanch for Pulsations in Planetary Nebulae Nuclei, (Ph D. Thesis: University of Texas at Austin).

Jensen, K. A., 1985, IAU Circ., No. 4102.

Jensen, K. A., Swank, J. H., Guinan, E. F., Sion, E. M., and Shipman, H. L. 1986, Ap. J. (Letters), 309, L27.

Nelson, B., and Young, A. 1970, P.A.S.P., 82, 699.

Robinson, E. L., Clemens, J. C., and Hine, B. P. 1988, Ap. J. (Letters), 331, L29. 